\title{
Microgenetic Research of the Multi-levelled Vocabulary Acquisition of English Writing Based on Production-Oriented Approach
}

\author{
Lijun Tao ${ }^{1, a}$, Yi Zhang 2,b,
}

1 Dalian Neusoft University of Information, Dalian, Liaoning, China

${ }^{2}$ Dalian Neusoft University of Information, Dalian, Liaoning, China

ataolijun@neusoft.edu.cn, bzhangyi@neusoft.edu.cn

${ }^{*}$ Corresponding author: Lijun Tao

Keywords: Production-Oriented Approach, microgenetic method, corpus, level of vocabulary, fossilization.

\begin{abstract}
The study researched the developmental features of vocabulary acquisition in English writing of Chinese university students on the basis of the Production-Oriented Approach (POA). The microgenetic method and corpus method were also used to serve the purpose. The researchers found that POA is viable in the teaching of college English writing; the multi-level teaching of vocabulary helps the students with a support and a stronger sense of multiplying their vocabulary; the microgenetic features of vocabulary in English writing indicate that teachers should render more help to students who cannot write effectively.
\end{abstract}

\section{基于 “产出导向型教学法”的英语写作词汇层次习得微变化研究}

\author{
陶立军1, a, 张翼 $2, b$, \\ 1 大连东软信息学院英语系, 沙河口区, 大连, 辽宁, 中国 \\ 2大连东软信息学院英语系, 沙河口区, 大连, 辽宁, 中国 \\ aemail: taolijun@neusoft.edu.cn, bemail: zhangyi@neusoft.edu.cn \\ 通讯作者:陶立军
}

关键词: 产出导向型教学法; 微变化; 语料库; 词汇层次; 石化

中文摘要：本研究基于 “产出导向型教学法” 理念, 使用微变化和语料库的研究方法, 探究 了大学英语写作中词汇习得的发展特征。研究结果发现: 该方法在大学英语写作教学中可行; 词汇层次教学能够使学生有清晰的词汇应用意识和依托; 微变化的写作词汇习得发展特征提 示教师应更多关注学生低谷状态的习得情况, 从而真正、全面提高学生的英语写作水平。

\section{1. 引言}

“产出导向型教学法（POA）”的理念由文秋芳教授在其 “输出驱动假设”（2007）和 “输出驱动一输入促成假设”（2014）的基础上，于2014年提出的较为完整的英语教学理论 体系。该体系包括教学理念、教学假设和以教师为中介的教学流程三大部分 (文秋芳, 2015), 其特征为教学流程由 “驱动”、“促成” 和 “评价” 三部分组成; 既强调产出过程, 又强调 产出结果；教师的中介作用体现在教学流程各个环节中。

随着POA理念的提出和大数据时代的到来, 国内外越来越多的专家和英语教育者已经开 始自主地将这种理念应用于英语教学和研究; 同时, 也有越来越多的英语学习者正在开始将 
这种方法作为辅助手段应用于自己的学习中。且不论成效如何, 单就其影响的范围和力度来 说, 这种 “本土化” 外语教学理论已不能忽视; 而且, 如何将这种方法系统地应用到教和学 的实践中去，已经成为摆在现代外语教育者面前的一个绕不开的问题; 或者可以这样说，该 理念所提出的手段和方法为大学英语写作的教学带来了新的契机（刘洁莹，2015）。

\section{2. 英语词汇教学}

词汇是英语学习的基础。但长期以来, 中国英语学习者包括大学生在语言输出端的词汇 能力整体上非常贫乏，其重要表现就是 “词汇石化” 现象（fossilization）。从写作词汇使用 的表现来看, 中国大学生在英语写作时, 较少主动或有效地使用最近学习过的词汇, 绝大多 数词汇还停留在初中或者高中较为简单的词汇阶段, 其结果就是语言无味、表现力差, 测试 中的表现是往往因为词汇的不当或不足使用而大幅度丢分。从深层次原因看, Selinker (1972) 将其定义为一种心理机制, 认为这种机制决定了学习者语言中的石化行为（文秋芳，2011）。

\section{3. 微变化的研究方法}

微变化研究法 (mecrogenetic method) 是近年来认知心理学领域中兴起的一种研究方法。 所谓微变化方法, 就是 “在重复多次的测试或观察的时间里, 给研究对象重复尝试同样的问 题或同类的问题，通过密集的观察，最终能够达到准确描述和了解发生着的变化（Miller \& Coyle，1999）。”该方法主要用于探究认知发展轨迹与机制, 侧重研究群体或个体发展过程 中的变异性（周丹丹，2012）。换句话说，该研究方法更加侧重 “过程” 的研究。据权威专 家预测, 这种动态的研究方法将最终成为一种不可或缺的主流研究方法。

\section{4. 研究综述及研究问题}

\section{1 英语写作词汇的 “石化” 现象}

Selinker（1972）最早提出 “石化” 的概念。1992年，他又把这个概念重新定义为 “学习 者中介语停止的过程, 表现为持续稳固的非目标与结构”。这样就将石化的定义扩展到最终 的语言获得（文秋芳，2011）。根据文秋芳教授的梳理，对于有关石化成因的解释主要有以 下几种：1）生物解释论，其代表人物Lenneberg（1967）等认为, 如果在语言关键期之前没 有发展相应的二语次系统, 那么语言习得很难有更大的进步; 2) 交互作用说, 该理论的提出 者Vigil和Oller (1976) 认为, 语言的石化不仅包括错误的语言形式而且包括正确的语言形式, 对语言石化现象起决定作用的因素是语用的方面的问题（陈慧媛，1999）。防止石化的理想 方法是肯定情感反馈和否定的认知反馈；3）文化适应模式, 提出者Schumann强调二语学习 者对目标语文化的接受程度决定了语言学习是否成功以及所能达到的水平。在写作教学实践 中，笔者也能强烈地感受到学生的词汇固话或石化的现象。

\section{2 关于英语写作词汇习得的研究}

英语写作词汇习得研究多数为实证研究，如聂文杰和李世民进行了用英语写作促进词汇 习得的实验, 发现教师对词汇使用的明确导向要求能够促进学生对词汇的应用和记忆(2016)。 刘晓树则认为: 对词汇广度和深度知识习得较好的学生更能有效提高英语写作水平（2013）。 褚艳 (2009) 认为词汇产出能力直接影响其作文质量, 其研究同时证明网络自主学习对学习 者的词汇习得及产出能力的积极作用。但是, 从以上的研究可以看出, 英语写作习得的输入 显然不是系统的, 这会造成学生在写作中对于词汇的应用意识和层次意识相应地弱化。 


\section{3 英语写作微变化研究现状}

根据动态系统理论 (dynamic systems theory) 的观点, 语言系统是一个复杂的动态系统, 语言包括二语习得具有动态的、复杂的和变异的特点。微变化研究法强调语言能力变化的复 杂性和不可预知性, 契合了动态系统理论的语言习得的观点（周丹丹，2012）。写作习得的 微变化研究国内很少, 但使用该方法的研究近几年有增加的趋势。李利英进行了英语写作动 态评估过程的微变化研究 (2015), 但研究对象为高中生。张燕敏进行了基于语料库的二语 写作语体词汇维度微变化研究（2016），但没有进行写作干预的专门指导环节。

\section{4 研究问题}

综上所述，在当前我国英语教学改革已进入深水区和英语教学已经进入大数据时代的背 景下, 我国的大学英语教学和研究尚处在传统英语模式和信息化手段并存的阶段, 亟需进行 可靠的理论创新和教学方法的研究与实践。本研究即是基于 “产出导向型教学法” 的理念, 重点研究以平台为手段的大学英语写作教学的词汇习得问题, 研究视角为微变化层面。本研 究的研究问题如下：1）在教师的指导下, 学生英语写作词汇呈现怎样的输出演进模式? 2) 写作词汇层次的输入指导能否起到预期的效果?

\section{5. 研究方法}

\section{1 研究平台}

本实验主要是基于英语写作批改平台句酷批改网来实施。

\section{2 被试者}

本实验选择参与本项目意愿较强的 30 名大二学生进行系统的微变化教学实验（为保证沟 通管理方便, 实验人员选择了来自同一专业的学生。该30名学生经本校开发的CASEC英语考 试系统测试, 成绩处于中上等), 教师以周为单位向学生布置写作任务并上传句酷批改网。

\subsection{POA教学法研究步骤与数据收集}

研究人员自行开发了基于各个层次的中国英语学习者的英语词库和写作词库。该词库按 照学习层次分为初中、高中和大学英语四级难度; 又将每个层次的高频词汇进行横向和纵向 汇总, 供课堂讲解和学生修改词汇使用（促成环节）。在教学指导环节, 教师规定: 同一高 频单词, 如果在同一篇作文使用超过了两次, 必须查询改写作词库, 使用同义词表达, 不允 许出现重复使用的情况。我们将词汇最高难度定在大学英语四级的主要考虑是：一，学生所 处的阶段; 二, 如能将大学四级难度的单词成功应用到写作中, 已经是较高水平的产出结果。

项目组教师对 30 名学生进行写作纸质前测, 以备后续研究使用。从第一周开始, 在课堂 上讲授本周写作任务, 使用相关写作素材与学生进行现象分析、观点讨论和逻辑整理, 重点 是本周写作任务可能用到的高频词汇和场景词汇（驱动环节）。每周写作部分授课结束后, 在批改网上为学生布置本周写作作业（长度为大学英语四级考试要求字数：120到180字）。

学生在批改网上完成作业后, 将平台反馈的分数和修改建议（评价环节）截图、导出作 文的电子版并进行修改（记录每次平台的词汇修改建议）。对于词汇搭配等方面的疑惑, 指 导学生查阅 “微软必应词典”（Bing Dictionary）英文单词解释; 如有进一步疑惑, 指导后者 查阅 “美国当代英语语料库 (COCA) ” 观察某词的前后共现搭配, 以彻底解除疑惑（这个 方法有些难度, 但考虑到学生主要是观察某个词的前后共现搭配, 难度不是很大）。如果还 有无法解决的词汇方面的用法, 学生将其记录下来, 通过 “微信” 或 “微信网页版” 本周内 向教师咨询。如有不认识的单词, 首先通过网络字典进行自学（促成环节）。在每周的写做 任务完成回收后, 教师对学生写作任务的整体问题进行课堂讨论讲解（评价环节）, 然后指 导学生将修改后的写作作业再次以电子版提交以巩固学习效果和观察教学效果。 
从第一周开始, 研究教师每周将学生的作文从批改完导出并存档, 本学期12周写作教学 结束后, 再布置一篇纸质写作作为后测 (为保证样本数量较为整齐, 研究人员人为控制了后 测字数, 方法是提前告知学生前测的字数, 在后测写作时将字数控制在前测基础上加减 5 个单 词的范围内）。教师整理本学期的学生作文建成第一学期微型研究语料库并根据研究需求进 行语料标注。稍后，根据观察结果，又使用 “问卷星” 对30个学生进行了问卷调查。

\section{4 研究工具}

平时的写作主要使用AntConc3.2.1w进行研究; 前、后测结果使用SPSS统计软件进行统计。

\section{6. 结果和讨论}

\section{1 前测和后测的对比分析}

为了对比本学期写作词汇层次习得的总体变化和本研究的教学效果, 我们将前后测的主 要考察结果进行了配对样本 $\mathrm{t}$ 检验。

表1 个人习作类符/形符比配对样本t检验结果

\begin{tabular}{c|c|c|c|c|c|c|c}
\hline \multicolumn{3}{|c|}{ 前测 } & \multicolumn{3}{c|}{ 后测 } & $\mathrm{t}$ & $\mathrm{p}$ \\
\hline $\mathrm{N}$ & $\mathrm{MD}$ & $\mathrm{SD}$ & $\mathrm{N}$ & $\mathrm{M}$ & $\mathrm{SD}$ & \multirow{2}{*}{-2.335} & \multirow{2}{*}{0.043} \\
\hline 10 & 0.3310 & 0.2885 & 10 & 0.3510 & 0.3281 & & \\
\hline
\end{tabular}

表1显示了受试学生前测和后测的类符/形符比。这个指标能够大体观察学生的用词丰富 程度。从检验结果看, 前后测的平均成绩为 0.3310 和 $0.3281, \mathrm{t}$ 值为 $-2.335, \mathrm{p}<0.05$ 。这说明经 过一学期的语言实验, 学生的用词丰富程度得到了显著提升。

研究人员也观察了前测后测词语使用错误（包括搭配、语体、词形变化、词性、拼写等） 的检测结果，毕竟，用词丰富是一个方面，能否正确使用是另一个方面（见下表）。

表 2 个人习作词语使用错误配对样本t检验结果

\begin{tabular}{c|c|c|c|c|c|c|c}
\hline \multicolumn{3}{|c|}{ 前测 } & \multicolumn{3}{c|}{ 后测 } & $\mathrm{t}$ & $\mathrm{p}$ \\
\hline $\mathrm{N}$ & $\mathrm{MD}$ & $\mathrm{SD}$ & $\mathrm{N}$ & $\mathrm{M}$ & $\mathrm{SD}$ & \multirow{2}{*}{2.753} & \multirow{2}{*}{0.022} \\
\hline 30 & 12.20 & 1.8738 & 30 & 10.60 & 2.5033 & \\
\hline
\end{tabular}

从表2可以看出, 受试的30位学生在整体上经过一个学期的学习, 词语使用错误下降幅度 较大 $(\mathrm{p}=0.022<0.05)$, 达到显著水平。但是标准差在后测中增大, 说明不同的学生的下降 幅度不一样。考察原始分数, 个别学生有写作错误有未下降的情况。

为了检验受试学生对不同层次词汇的掌握程度, 笔者对三个层次的单词使用情况进行了 考察, 结果如下:

表3 三个学习层次词汇变化结果 (使用配对样本t检验)

\begin{tabular}{c|c|c|c|c|c|c|c|c}
\hline \multirow{2}{*}{ 单词层次 } & \multicolumn{4}{|c|}{ 前测 } & \multicolumn{3}{|c|}{ 后测 } & \multirow{2}{*}{$\mathrm{t}$} \\
\cline { 2 - 8 } & $\mathrm{N}$ & $\mathrm{MD}$ & $\mathrm{SD}$ & $\mathrm{N}$ & $\mathrm{M}$ & $\mathrm{SD}$ & & $\mathrm{p}$ \\
\hline 初中 & 30 & 116.4 & 9.7661 & 30 & 100.9 & 10.2464 & 3.345 & 0.009 \\
\hline 高中 & 30 & 25.00 & 4.6188 & 30 & 35.60 & 11.4523 & -2.939 & 0.017 \\
\hline 四级 & 30 & 0.70 & 1.0594 & 30 & 6.00 & 2.7080 & -5.618 & 0.000 \\
\hline
\end{tabular}

表3表明, 在后测中, 受试学生使用初中水平的英语单词的数量大大减少 $(\mathrm{p}=0.009<0.05$, 与前测相比, 差异显著）, 而高中英语单词的使用显著增加（ $\mathrm{p}=0.017<0.05 ）$ 。以上结果表 明, 在本研究中, 教师对于学生的明确的词汇层次的指导取得了明显的效果。然而, 后测中 大学四级水平的词汇的增长虽然也达到了显著水平, 但是, 考察原始成绩和表中平均值, 这 个数量与作文总字数相比还是很小的, 远未达到实验教师的理想状态。 


\section{2 历次写作微变化分析}

在写作词汇的微变化研究层面, 研究人员关注的是每个学生同样的维度中的变化过程, 即: 类符/形符比、词语使用错误、三个层次上的单词的使用比例变化。由于微变化的结果繁 杂, 图表众多, 不方便在此一一列出（详细的表格仅供计算结果和作与学生沟通反馈之用）, 笔者谨举出一名较为有代表性的学生（以下称S3）的例子，足可窥全貌。

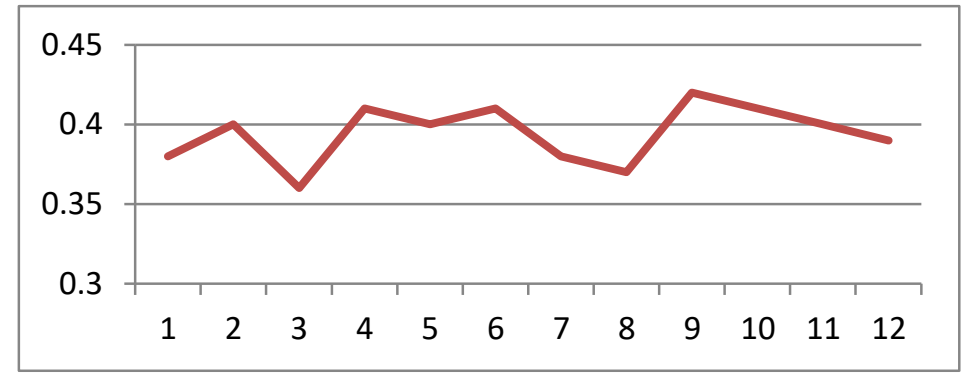

图1 S3个人习作类符/形符比各次变化情况

从图1可以看出, S3 的写作类符/形符比呈现高低起伏状态, 但经过与该生前后测的该项 指标对比后（分别为0.37和0.39）可以发现，总体上，该生的这项指标呈上升状态。

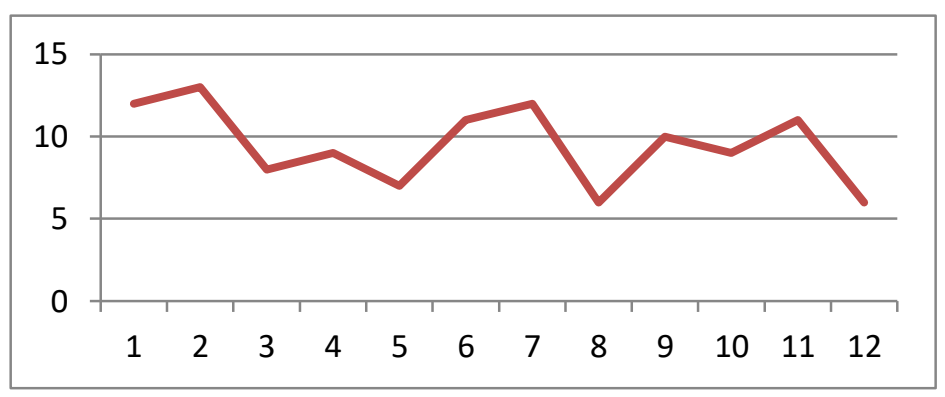

图2 词语使用错误变化趋势

图2表明, 该生的词语使用错误的微变化也呈不规则的趋势, 与前测相比 (11个), 该生 写作过程中的词语错误数量有升有降, 有的词语错误已经降到了 6 个, 但是, 该生在后测中的 该项指标为 10 个。与前测比, 这个降幅并不明显, 说明该生的语言基础, 尤其是词汇意义或 理解方面仍然薄弱。

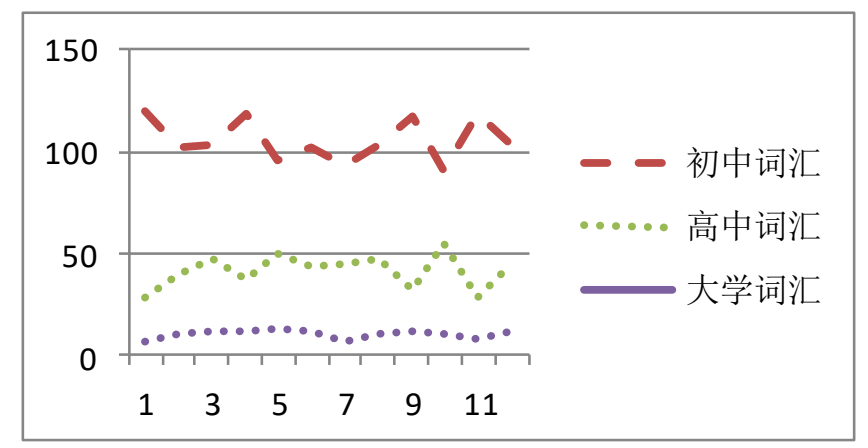

图3 S3 三个学习层次词汇变化结果

根据图3显示，S3在整个实验过程中的总体趋势是：初中词汇占多数，其次为高中词汇， 再次为大学四级词汇。然而, 与其前测成绩 (分别是 $127 、 30 、 3$ ) 和后测成绩 (分别是97、 53、7）相比，图中的结果比较接近该生的真实的写作中的词汇水平。 


\section{7. 结语}

本实验结合大学英语教学实践, 证实了 “产出导向型教学法” 与大数据的综合方法在大 学英语写作教学中的可行性。写作词汇教学的分层方法也较为有针对性和创新性; 微变化的 研究方法观察到了学生的波浪式的进步过程。但是, 本研究也存在部分不足, 如, 本研究的 受试学生较多, 对于学生的更多的写作细节如学生的什么词性习得更加容易、学生在写作具 体的主题稍难的篇章时的心理和行为过程如何影响词汇选择、男女生在写作词汇微变化的诸 方面是否会呈现不同的趋势等方面都没有关注，这些都应该是以后词汇研究应该探讨的问题。

\section{致谢}

本文为北京外国语大学中国外语与教育研究中心第八批中国外语教育基金项目《产出导 向型教学法” 在大学英语写作教学中的应用研究——微变化视角》（项目编号：W2013318） 及教育部职业院校外语类专业教学指导委员会基于大数据的高职英语写作教学创新研究课题 《基于大数据的英语写作习得微变化研究》（项目编号：GZGZ5414-18）的阶段性成果之一。

\section{References}

[1] Chen Qingbin, Reconstruction of the Teaching Model of College English Writing in the Big Data Era, Foreign Language Research, vol. 3, pp. 130-131, 2016.

[2] Chu Yan, College English Writing Teaching Mode Based on Autonomous Online Learning, Journal of Ningbo University of Technology, vol. 2, pp. 56-58, 2009.

[3] Li Lijun, Yu Han and Guo Qi, A Review of POA in Research, Wenjiaoziliao, vol. 8, pp. 167-169, 2017.

[4] Liu Jieying, College English Writing Assessment in the Big Data Era, Intelligence, vol. 34, pp. 10-11, 2014.

[5] Liu Zuoxiong, Fossilization of Vocabulary in English Writing, Journal of Southwest Minzu University, vol. 12, pp. 155-157, 2007.

[6] Liu Xiaoshu, Empirical Research of Acquisition in Lexical Knowledge Affecting English Writing, Journal of Jixi University, vol. 3, pp. 75-76, 2013.

[7] Nie Wenjie and Li Shim, Empirical Research of Vocabulary Acquistion by Way of English Writing on the Basis of Teaching of English Majors in Application-Oriented University, Teaching Forum, vol. 2, pp. 275-276, 2016.

[8] Qian Wenjuan, A New Model of Automatic Scoring of English Writing for Higher Vocational Students in the Big Data Era, Journal of Kaifeng Institute of Education, vol. 10, pp. 175-176, 2014.

[9] Wang Haixiao, Reform in the Teaching of College English Writing in the Big Data Era, Modern Distance Education Research, vol. 3, pp. 66-72, 2014.

[10] Wen Qiufang, Major Issues in Second Language Acquisition, Foreign Language Teaching and Research Press, 81-82, 2011.

[11]Wen Qiufang, Constructing the Theoretical Framework of the Production-Oriented Approach, Foreign Language Teaching and Research, vol. 4, pp. 547-558, 2015.

[12]Zhang Yanmin, On the Micro-variations in Lexical Dimensions of Genre in Longitudinal Written Productions of EFL Learners, Journal of Qiqihar University, vol. 12, pp. 128-130, 2016. 
[13]Zhang Wenjuan, Experimental Study of the Influence of POA on College English Writing, Modern Foreign Languages, vol. 3, pp. 377-385, 2017.

[14]Zhou Dandan, Microgenetic Method in Applied Linguistics, 1-7, 2012. 